\title{
Defects Formation and Amorphization of Zn-MOF-74 Crystals by Post-Synthetic Interactions with Bidentate Adsorbates
}

\author{
Jonathan B. Lefton, ${ }^{a}$ Kyle B. Pekar, ${ }^{a}$ Uroob Haris, ${ }^{a}$ Mary E. Zick, ${ }^{b}$ Phillip J. Milner, ${ }^{b}$ Alexander R. \\ Lippert, ${ }^{a}$ Ljupčo Pejov, ${ }^{c}$ Tomče Runčevski*a \\ The controlled introduction of defects into MOFs is a powerful strategy to induce new physiochemical properties and \\ improve their performance for target applications. Herein, we present a new strategy for defect formation and \\ amorphization of the canonical MOF-74 frameworks based on fine-tuning of adsorbate-framework interactions in the metal \\ congener, hence introducing structural defects. Specifically, we demonstrate that controlled interactions between the MOF \\ and bidentate ligands adsorbed in the pores initiates defect formation and eventual amorphization of the crystal. These \\ structural features unlock properties that are otherwise absent in the ordered framework, such as broad-band fluorescence. \\ The ability to introduce defects by adsorbate-framework interactions, coupled with the inherent tunability and modularity \\ of these structures, provides a new route for the synthesis of diverse heterogeneous and hybrid materials.
}

\section{Introduction}

Metal-organic frameworks (MOFs) are porous materials with promising applications owing to their permanent porosity, high surface area, and structural tunability. The directional and dynamic nature of the interactions between the metal ions and organic linkers enable the growth of crystalline structures with pronounced structural order and periodicity. ${ }^{1}$ The crystal structures of MOFs, like all solid materials, ${ }^{2}$ deviate from longorder periodicity and contain defects, disruptions and irregularities. The presence of defects and/or amorphous regions does not necessarily have adverse effects on the properties of the materials; in fact, the controlled introduction of defects through "defect engineering" can increase performance and introduce new properties. ${ }^{3}$ For example, defects in MOFs have been shown to enhance gas adsorption, ${ }^{4 a, b}$ catalytic activity, ${ }^{4 b, c, d}$ proton conductivity, ${ }^{4 e}$ and mechanical response..$^{4 \mathrm{f}}$ The extreme case of defective MOFs are amorphous frameworks that exhibit a complete absence of long-order periodicity. ${ }^{5}$ Amorphous MOFs have been successfully used for targeted drug delivery, ${ }^{6 a}$ capture of harmful pollutants, ${ }^{6 b}$ functional glasses, and composite materials. ${ }^{6 c}$

The MOF-74 series of frameworks (Figure $1 a)^{7}$ is constructed from the 2,5-dioxido-1,4-benzenedicarboxylate (dobdc ${ }^{4-}$ ) linker ${ }^{7 a}$ and various divalent metals $\left(\mathrm{Mg}^{2+}, \mathrm{Mn}^{2+}, \mathrm{Fe}^{2+}, \mathrm{Co}^{2+}, \mathrm{Ni}^{2+}\right.$, $\mathrm{Cu}^{2+}, \mathrm{Zn}^{2+}$ and $\left.\mathrm{Cd}^{2+}\right)$. Related linkers such as extended congeners, $^{7 \mathrm{~b}}$ meta-substituted isomers, ${ }^{7 \mathrm{c}}$ and variants with heteroatoms on the core ${ }^{7 d, e}$ or on the terminal groups, ${ }^{7 f, 8}$ provide MOFs with the same topology, establishing a large family of functional frameworks. Furthermore, different combinations of linkers and metals within the same structure enable the preparation of multivariate MOF- $74 .{ }^{8}$ Despite the widespread study of MOF-74 materials, reports of defective or amorphous analogues remain relatively scarce.9,10 The propensity for defect formation is often related to the flexibility of coordination solids, 11 and the MOF-74 structure exhibits a high degree of connectivity, coupled with a low degree of flexibility, that may hinder defect formation and controlled amorphization. However, recent reports indicate that adsorbate-induced, structural distortions are possible in MOF$74 .{ }^{12}$ Here we explore the possibility of fine-tuning adsorbateframework interactions to move beyond subtle distortion and introduce structural defects in MOF-74 frameworks and, eventually, amorphization of the crystals.

In the crystal structure of activated MOF-74 (Figure 1a), there are pairs of neighbouring open metal sites that can be bridged by bidentate adsorbate molecules of fitting size and shape (Figure 1b). If the length of the adsorbate is slightly shorter than the optimal distance between two open metal sites, the bidentate molecule is unable to simultaneously bind to both cations and maintain a stable coordination environment. Typically, such adsorbents would bind with one end on the open metal site, and adopt a relative orientation pointing towards the middle of the pore. We hypothesized that, if the bidentate adsorbent has strong tendency to bind to the metal cation (such as thiol-functionalized adsorbents interacting with open $\mathrm{Zn}^{2+}$ sites), the bidentate adsorbate will effectively pull the cations away from the framework in order to simultaneously bind to both neighbouring sites, thus producing local defects in the form of structural displacements of the metal congeners. The grafting of 1,2-ethanedithiol (EDT) to $\mathrm{Zn}_{2}$ (dobdc) represents an ideal platform to test the strategy of adsorbate-induced introduction of such defects. This is because the thiol functional groups in EDT should strongly bind to the open site of the soft $\mathrm{Zn}^{2+}$ cation, but the length of EDT ( $\sim .6 \AA)$ is slightly shorter $(\sim 0.8 \AA)$ than the optimal distance ( $\sim 5.4 \AA)$ for a dithiol molecule to bridge two neighbouring cations in $\mathrm{Zn}_{2}$ (dobdc) along the plane of the pore (Figure $1 \mathrm{~b}$ ). Therefore, simultaneous binding of EDT to two neighbouring metal sites should displace the $\mathrm{Zn}^{2+}$ cations (Figure 1c) and introduce structural dislocations. 
a

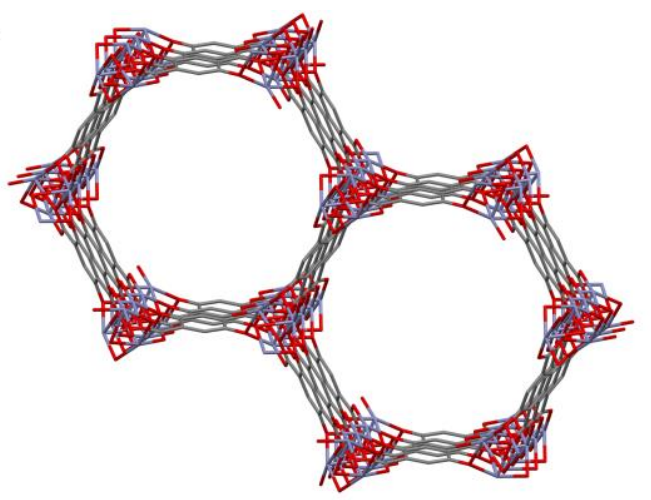

b

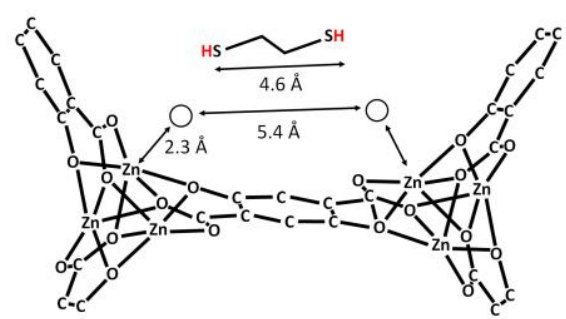

C

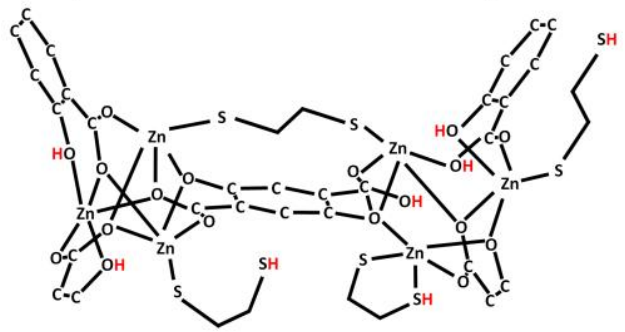

Figure 1. a) One pore of desolvated $\mathrm{Zn}_{2}$ (dobdc). Schematic representation of the b) connectivity in ordered $\mathrm{Zn}_{2}$ (dobdc), showing the optimal distance between the adsorption sites of two neighbouring $\mathrm{Zn}$ cations, and c) disruption of the local structure of the MOF upon adsorption of bidentate ligand.

\section{Results and Discussion}

To study the formation of defects using this strategy, we gradually increased the loading of EDT in $\mathrm{Zn}_{2}$ (dobdc) (see the SI for further details) and monitored the changes in crystallinity of the sample by powder X-ray diffraction (PXRD). As expected, the PXRD patterns (Figure 2a) show gradual loss of crystallinity, and eventual amorphization of the crystals, upon exposure to higher concentrations of EDT in methanol. The corresponding IR spectra (Figure $2 \mathrm{~b}$ ) of the samples feature a vibrational band centred at $2822 \mathrm{~cm}^{-1}$, assigned to the $\mathrm{sp}^{3} \mathrm{C}-\mathrm{H}$ stretching vibration of the EDT, ${ }^{13}$ confirming the incorporation of the adsorbate in the MOF crystals. The incorporation of EDT was also confirmed by thermogravimetric analysis (Figure 2c), and in fact increased the thermal stability of the samples. As a control experiment, we repeated these grafting experiments with $n$ - propyl mercaptan (nPM) as a monodentate adsorbate of similar size as EDT. Adsorption of nPM did not lead to observable defect formation or amorphization. In fact, the nPM-loaded sample maintained sufficient crystallinity to allow for structural determination. The crystal structure was solved from PXRD data and refined using the Rietveld method. ${ }^{14}$ In the crystal structure, every $\mathrm{Zn}^{2+}$ cation is grafted with a nPM molecule (see Figure S4) with the hydrocarbon part of the adsorbate molecule pointing towards the centre of the pores. Further structural details are provided in the SI.

Elucidating the local structure of the defective and/or amorphous regions in EDT- $\mathrm{Zn}_{2}$ (dobdc) crystals is not trivial. Conventional crystallographic methods are not appropriate due to loss of long-range order. Techniques that probe the local structure are also challenging because every metal site in the structure can be uniquely defected and surrounded by a coordination sphere with different local structure. To better understand the possible local geometries, we performed theoretical modelling of fragments of the MOF structure (see SI for further details). In order to mitigate surface effects, we extended the size of the model clusters and included 1968 atoms. The calculations were carried out at density functional tight binding (DFTB) level of theory with the DFTB+ code. ${ }^{15}$ Most of the EDT adsorbates were placed in close proximity to one open metal site, some of the metal sites were left open, and one EDT molecule was set in close proximity to two neighbouring sites. The calculations indicate that upon adsorption of EDT on a single open metal site, the $\mathrm{Zn}^{2+}$ atom slightly changes its position, moving closer to the adsorbate. This dislocation does not change the coordination sphere around the metal and has been observed in numerous crystal structures of MOF-74. ${ }^{7}$ On the contrary, the EDT molecule simultaneously binding two open sites has a strong "pulling" effect and dislocates the cations, changing their local geometry (Figures S9).

The dislocation of $\mathrm{Zn}^{2+}$ cations triggers reorganization of the surrounding linkers which adapt to the new local geometry. As such, defect formation on a single metal node will inevitably influence the local geometry of the neighbouring metal centres, perpetuating structural changes throughout the framework. This perpetuating mechanism of defect formation is suggested by the PXRD results. Specifically, upon gradual loading, the Bragg peaks incur a reduction in intensity, but maintain similar peak shape (Figure S6). This observation suggests that structural defects are not evenly distributed within the crystal. Instead, we hypothesize that the microcrystals consist in part of defected/amorphous regions, and in part of unaffected crystalline regions that afford the Bragg peaks of MOF-74.

The amorphous regions may exist as an integral part of the MOF sample, in a form of surface coating or patches distributed throughout the bulk of the crystal, or as separate solid particles that would co-precipitate upon decomposition or digestion of the MOF crystals. In order to observe the defected or amorphous regions within the microcrystals, we performed scanning electron microscopy (SEM) and energy dispersive $X-$ ray (EDX) analysis on a highly defected sample, corresponding to $100 \%$ EDT loading. The PXRD pattern of the sample (Figure 2a) exhibited an absence of Bragg reflections, indicative of 

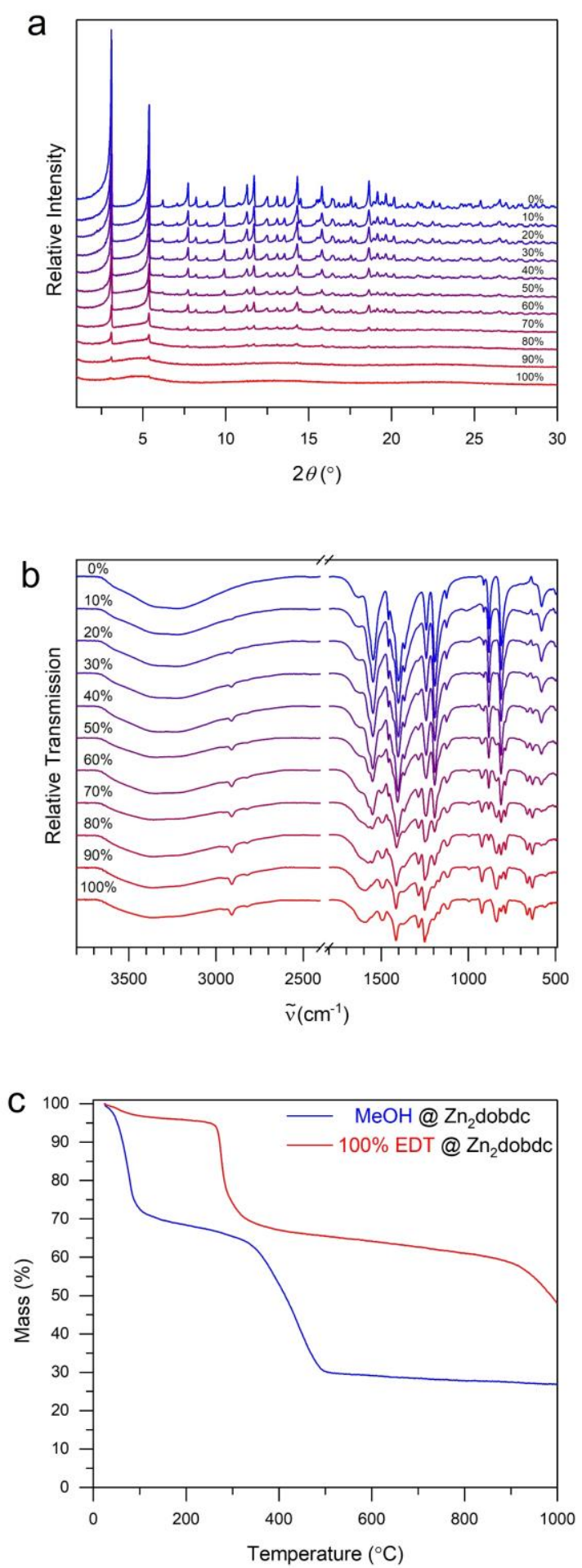

Figure 2. a) Powder diffraction patterns $(\lambda=0.70930 \AA)$ and b) infrared spectra of $\mathrm{Zn}_{2}$ (dobdc) as a function of EDT loading. c) Thermogravimetric curves of two representative samples. amorphous materials. The EDX analysis (Figure 3 ) proved a homogeneous distribution of oxygen, sulphur and zinc, indicating that EDT adsorbates are evenly distributed throughout the sample. Interestingly, the brightfield images of the sample show solid particles with sharp edges, with shape and size resembling those of MOF microcrystals. We hypothesize that such particle shape cannot result from precipitation of amorphous material, but it is "inherited" from the MOF microcrystals that were gradually defected by the EDT adsorbates. In fact, in the structure of MOF-74, each cation is connected to four linker molecules, and each linker connects eight metal cations. The high degree of connectivity affords the framework with a capacity to tolerate a substantial number of defects without deteriorating or dissolving. In order for a linker to be cleaved and removed from the framework, all eight coordination bonds must be broken, whereas a single EDT molecule can replace only two oxygen atoms. Our theoretical modelling shows that in the event of a complete removal of the dobdc ${ }^{4-}$ linker, two EDT molecules can occupy the gap and effectively "stitch" the framework, preventing digestion by the EDT adsorbates (see Figure S10).

The chemical composition of the highly defected samples is expected to change, with a substantial part of the dobdc ${ }^{4-}$ linkers eventually being replaced by EDT adsorbates. To assess the amount of ligand exchange, nuclear magnetic resonance (NMR) spectra were collected at different loadings and the concentration of the linker was followed in the solution (see the SI for further details). These results show an upper limit of $66 \%$ linker exchange reached at $75 \%$ dosing of EDT/Zn. Higher dosing of EDT did not result in an increase of the ligand exchange, indicating a stable composition of the amorphous regions represented with a $\mathrm{Zn}_{6}$ (dobdc)(EDT) ${ }_{2}$.

In addition to the dislocation of zinc cations, grafting of EDT to the framework would inevitably lead to the breaking of O$\mathrm{Zn}^{2+}$ bonds, allowing exposed oxygen atoms to become protonated by the EDT molecule. In fact, our modelling studies show that such a proton transfer is possible and may even

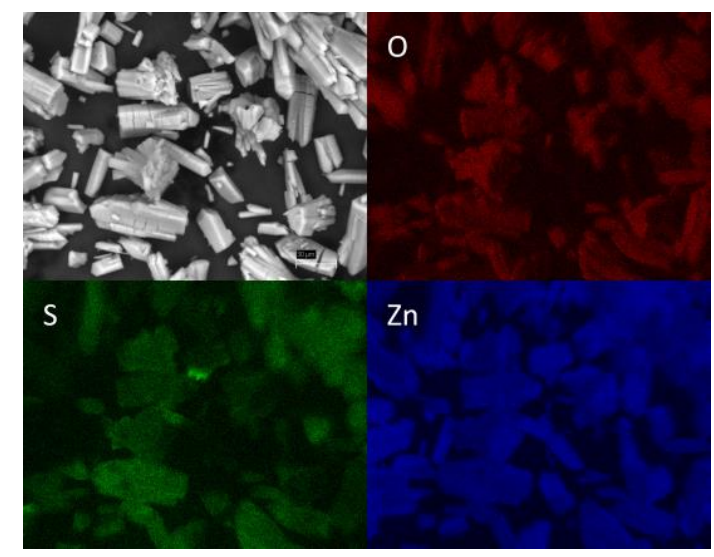

Figure 3. Brightfield SEM image and EDX color-coded elemental distribution of EDT-loaded $\mathrm{Zn}_{2}$ (dobdc) at $100 \%$ loading. 
precede the $\mathrm{Zn}^{2+}$ dislocation. The flexible and dangling thiol groups of a bound EDT molecule on a single site can reach a neighbouring atom and initiate a proton transfer (histograms of $\mathrm{SH} \cdots \mathrm{O}$ are given in Figures S11 and S12). The lack of a noticeable IR band in the spectral region from 2500 to $2600 \mathrm{~cm}^{-1}$ in the IR spectra of EDT loaded samples, indicative of $\mathrm{S}-\mathrm{H}$ vibrations, ${ }^{13}$ (Figure $2 \mathrm{~b}$ ) supports the hypothesis for deprotonation of the adsorbate molecules.

Protonation of the dobdc- linker enables us to observe the defected regions by measuring the photoluminescence of the protonated linker. Protonated variants of the dobdc ${ }^{4-}$ linker exhibit photoluminescence due to excited-state intramolecular proton transfer (ESIPT) between the phenol and carboxylic oxygen atoms, typical for salicylic acid derivatives. ${ }^{16}$ Figure $4 a$ presents samples of as-synthesized, nPM-loaded, and EDTloaded $\mathrm{Zn}_{2}$ (dobdc) under UV (365 $\mathrm{nm}$ ) irradiation. The methanol-solvated and nPM-loaded MOFs do not demonstrate photoluminescence, as expected for frameworks made of fully deprotonated linkers; however, the defected framework shows bright yellow-green light emission. The corresponding photoluminescence (PL) spectra are presented in Figure $4 \mathrm{~b}$ and show a broad-band absorption and emission centred at $368 \mathrm{~nm}$ and $510 \mathrm{~nm}$, respectively. The light absorption is attributed to transitions between singlet $\pi-\pi^{*}$ excited states of the protonated fragment of the ligand, similar to MOFs made of $\mathrm{H}_{2}$ dobdc $^{2-}$ ligands. ${ }^{17}$ The large Stokes shift of $142 \mathrm{~nm}$ further corroborates the hypothesis that fluorescence is due to ESIPT. ${ }^{16 b}$ Expectedly, the emission intensity was found to increase with the amount of defects in the framework. Figure $4 c$ presents the fluorescent intensity as a function of EDT loading. The graph shows a linear dependence of the fluorescence intensity with the EDT concentration below a loading of $70 \% \mathrm{EDT} / \mathrm{Zn}$, followed by a plateau at higher concentrations. The observation of saturation at high loading is consistent with the proposed mechanism of defect formation. The bidentate EDT adsorbate can simultaneously bind two $\mathrm{Zn}^{2+}$ cations; at low concentration of EDT, each adsorbate can theoretically reach to two neighbouring open metal sites, form a defect and contribute to an increase of the fluorescence. At higher loadings, a substantial portion of the open metal sites is already coordinated by adsorbates and proton transfer has occurred. As a control experiment, we performed the same loading procedure with nPM. Expectedly, higher loadings of nPM did not result in an increase in fluorescence, confirming that a bidentate dithiol is required to systematically introduce defects (Figure 4c).

Fluorescent materials can be used as on-off sensors of metals based on the quenching of fluorescence through nonradiative decay pathways; indeed, MOFs have been extensively studied in this regard. ${ }^{18}$ One of the major challenges of metal sensors is a low selectivity, as various paramagnetic ions can quench the fluorescence. The light emitting centres in EDT loaded $\mathrm{Zn}_{2}$ (dobdc) are embedded in a thiol-rich environment and surrounded by $\mathrm{Zn}^{2+}$ cations with various local geometries. This structural environment has different affinity to metals, thereby imparting selectivity to the framework; different cations have different degrees of penetration in the framework a

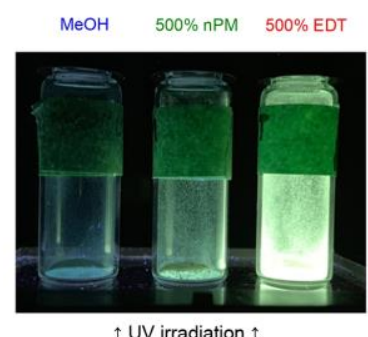

$\uparrow$ UV irradiation $\uparrow$
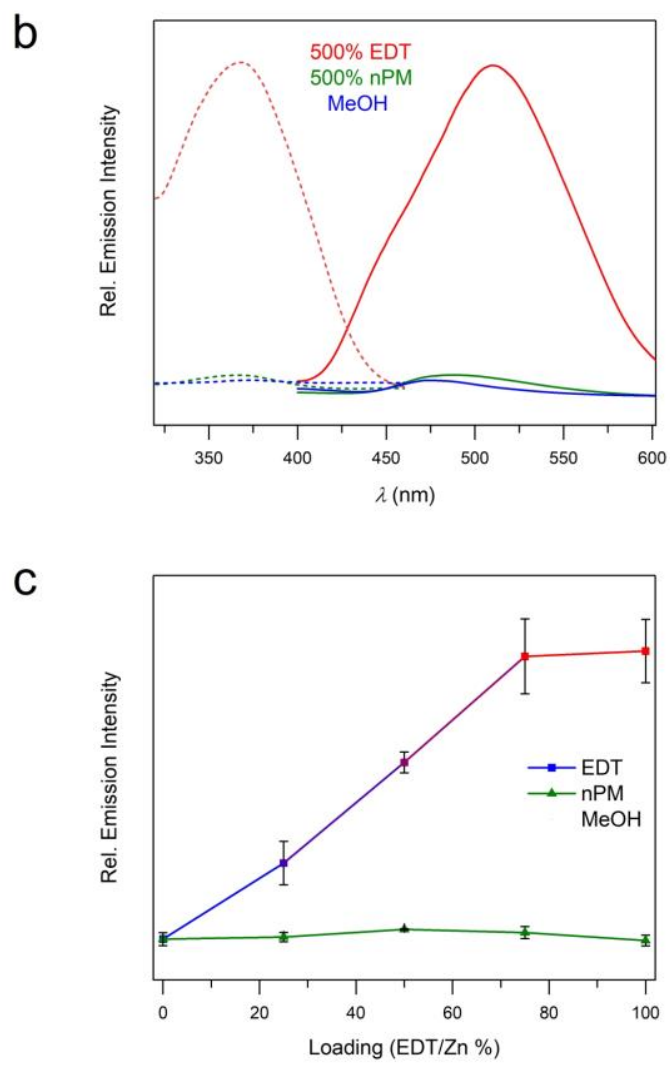

Figure 4. a) Photographs of methanol-solvated, nPM-loaded, and EDT-loaded $\mathrm{Zn}_{2}$ (dobdc) powder under $365 \mathrm{~nm}$ irradiation. b) Photoluminescence spectra of the samples showing excitation (dashed) and emission (solid). c) Change in the fluorescence intensity of EDT-loaded $Z n_{2}$ (dobdc) as a function of EDT concentration (blue-to-red lines) and $\mathrm{nPM}$-loaded $\mathrm{Zn}_{2}$ (dobdc) as a function of nPM concentration (green lines). The lines in the graph are included to guide the eye.

and the embedded protonated linkers. We tested the EDT loaded materials as a potential sensor for metal ions in aqueous solutions (see the SI for further details). Figure 5 presents the changes in the fluorescence after $1 \mathrm{~h}$ soaking in a $0.1 \mathrm{M}$ solution of fifteen different cations. We observe selectivity towards $\mathrm{Ag}^{+}$, followed by $\mathrm{Fe}^{3+}$ and $\mathrm{Au}^{3+}$. Further experimental and theoretical studies directed towards elucidation of the sensing mechanism are in progress. 


\section{Conclusions}

In summary, we show that post-synthetic adsorption of specific bidentate molecules in the pores of MOF-74 can lead to formation of structural defects and ultimately amorphization of the material. Our theoretical structural analyses indicate that the material can simultaneously possess metal centres with various local geometries, potentially including Lewis acidic sites, and Brønsted acidic sites on protonated linkers. Remarkably, in our experiments aimed at defecting $\mathrm{Zn}_{2}$ (dobdc) with EDT adsorbates, the overall size and shape distribution of the MOF crystal was retained upon defect introduction. The protonation of the linker allowed for emergence of new physicochemical properties, such as photoluminescence with broad-band emission. Presumably owing to the dense, thiol-rich hybrid structure, only certain metal cations were able to reach the embedded light emitters and thereby quench the fluorescence. This new method allows for controlled introduction of defects in MOF-74 materials; the general idea, however, is applicable to various MOFs featuring open metal sites and can be used in the design of advanced defective materials for a myriad of potential applications.
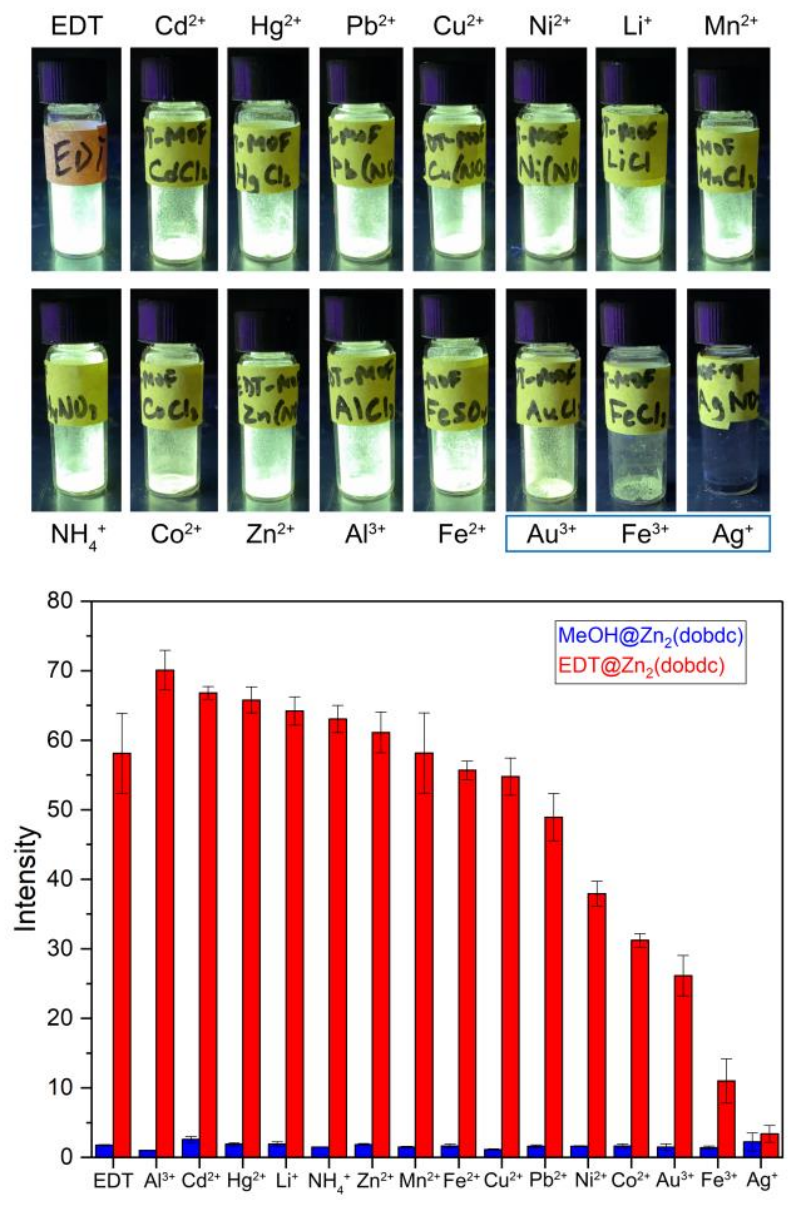

Figure 5. a) Dry samples of EDT loaded $\mathrm{Zn}_{2}$ (dobdc) after 24 soaking in solution of metal cations. b) Graph of the fluorescence intensity of the samples (red bars). The corresponding results of a control experiment with methanol solvated $\mathrm{Zn}_{2}$ (dobdc) are presented as blue bars.

\section{Conflicts of interest}

A.R.L. discloses a financial stake in BioLum Sciences, LLC, a company developing medical devices. P.J.M is listed as an inventor on several provisional patents involving functionalized variants of MOF-74.

\section{Acknowledgements}

We thank the Welch Foundation (Grant No.: N-2012-20190330) for financial support. K.B.P. thanks the Hamilton Fellowship. We thank Roy Beavers for his assistance with SEM imaging. We acknowledge Cornell University for financial support of M.E.Z. and P.J.M.

\section{Notes and references}

‡ Footnotes

1 B. F. Hoskins and R. Robson, J. Am. Chem. Soc., 1999, 112, 1546-1554.

2 C. P. Goodrich, A. J. Liu and S. R. Nagel, Nature Phys., 2014, 10, 578-581.

3 a) Z. Fang, B. Bueken, D. E. De Vos and R. A. Fischer, Angew. Chem. Int. Ed., 2015, 54, 7234-7254, b) D. S. Sholl and R. P. Lively, J. Phys. Chem. Lett., 2015, 6, 3437-3444, c) J. Ren, M. Ledwaba, N. M. Musyoka, H. W. Langmi, M. Mathe, S. Liao and W. Pang, Coord. Chem. Rev., 2017, 349, 169-197, d) S. Dissegna, K. Epp, W. R. Heinz, G. Kieslich and R. A. Fischer, Ad. Mater., 2018, 30, 1704501.

4 a) Y.-B. Zhang, H. Furukawa, N. Ko, W. Nie, H. J. Park, S. Okajima, K. E. Cordova, H. Deng, J. Kim and O, M. Yaghi, J. Am. Chem. Soc., 2015, 137, 2641-2650, b) O. Kozachuk, I. Luz, F. X. Llabrés i Xamena, H. Noei, M. Kauer, H. B. Albada, E. D. Bloch, B. Marler, Y. Wang, M. Muhler and R. A. Fischer, Angew. Chem. Int. Ed., 2014, 53, 7058-7062, c) G. Cai and H-L. Jiang, Angew. Chem. Int. Ed., 2017, 56, 563-567, d) W. Wang, D. I. Sharapa, A. Chandresh, A. Nefedov, S. Heißler, L. Heinke, F. Studt, Y. Wang and C. Wöll, Angew. Chem. Int., Ed., 2020, 59, 1-6, f) J. M. Taylor, S. Dekura, R. Ikeda and H. Kitagawa, Chem. Mater., 2015, 27, 2286-2289, g) S. Dissegna, P. Vervoorts, C. L. Hobday, T. Düren, D. Daisenberger, A. J. Smith, R. A. Fischer and G. Kieslich, J. Am. Chem. Soc., 2018, 140, 11581-11584.

5 T. D. Bennett and A. K. Cheetham, Acc. Chem. Res., 2014, 47, 1555-1562.

6 a) C. Orellana-Tavra, E. F. Baxter, T. Tian, T. D. Bennett, N. K. H. Slater, A. K. Cheetham and D. Fairen-Jimenez, Chem. Commun., 2015, 51, 13878-13881, b) T D. Bennett, P. J. Saines, D. A. Keen, J.-C. Tan and A. K. Cheetham, Chem. Eur. J., 2013, 19, 7049-7055, c) T. D. Bennett, J.-C. Tan, Y. Yue, E. Baxter, C. Ducati, N. J. Terrill, H. H.-M Yeung, Z. Zhou, W. Chen, S. Henke, A. K. Cheetham and N. Greaves, Nature Commun., 2015, 6:8079.

7 a) N. L. Rosi, J. Kim, M. Eddaoudi, B. Chen, M. O'Keeffe and O. M. Yaghi, J. Am. Chem. Soc., 2005, 172, 1504-1518, b) T. M. McDonald, W. R. Lee, J. A. Mason, B. M. Wiers, C. S. Hong and J. R. Long, J. Am. Chem. Soc., 2012, 134, 7056-7065, c) M. T. Kapelewski, T. Runčevski, J. D. Tarver, H. Z. H. Jiang, K. E. Hurst, P. A. Parilla, A. Ayala, T. Gennett, S. A. FitzGerald, C. M. Brown and J. R. Long, Chem. Mater., 2018, 30, 8179-8189, d) D. J. Levine, T. Runčevski, M. T Kapelewski, B. K. Keitz, J. Oktawiec, D. A. Reed, J. A. Mason, H. Z. H Jiang, K. A. Colwell, C. M. Legendre, S. A. FitzGerald and J. R. Long, J. Am. Chem. Soc., 2016, 138, 10143-10150, e) R. W. Flaig, T. M. Osborn Popp, A. M. Fracaroli, E. A. Kapustin, M. J. Kalmutzki, R. M. Altamimi, F. Fathieh, J. A. Reimer and O. M. Yaghi, J. Am. Chem. Soc., 2017, 
139, 2125-12128, f) L. Sun, T. Miyakai, S. Seki and M. Dincă, J. Am. Chem. Soc., 2013, 135, 8185-8188. g) T. Runčevski, M. T. Kapelewski, R. M. Torres-Gavosto, J. D Tarver, C. M. Brown and J. R. Long, Chem. Commun., 2016, 52, 8251-8254.

8 a) L. J. Wang, H. Deng, H. Furukawa, F. Gándara, K. E. Cordova, D. Peri and O. M. Yaghi, Inorg. Chem., 2014, 53, 5881-5883, b) J. D. Howe, C. R. Morelock, Y. Jiao, K. W. Chapman, K. S. Walton and D. S. Sholl, J. Phys. Chem. C, 2017, 121, 627-635, c) B. L. Suh, S. Lee and J. Kim, J. Phys. Chem. C., 2017, 121, 24444-24451.

9 a) J. A. Villajos, N. Jagorel, S. Reinsch and F. Emmerling, Frontiers Matter., 2019, 6:230, b) N. E. A. El-Gamel, E. J. Inorg. Chem., 2015, 8, 1351-1358, c) D. Wu, W. Yan, H. Xua, E. Zhang and Q. Li, Inorg. Chim. Acta, 2017, 460, 93-98.

10 a) P. Ma, F. Meng, N. Wang, J. Zhang, J. Xie and B. Dai, ChemistrySelect, 2018, 3, 10694-10700, b) B. Li and H. C. Zeng, Chem. Mater., 2019, 31, 5320-5330, c) T. Zhang, J. Wang, W. Zhang, C. Yang, L. Zhang, W. Zhu, J. Sun, G. Li, T. Lid and J. Wang, J. Mater. Chem. A, 2019, 7, 2845-2854, d) S. Muratović, B. Karadeniz, T. Stolar, S. Lukin, I. Halasz, M. Herak, G. Mali, Y. Krupskaya, V. Kataev, D. Žilić and K. Užarević, J. Mater. Chem. C, 2020, 8, 7132-7142.

11 a) T. D. Bennett, A. K. Cheetham, A. H. Fuchs and F.-X. Coudert, Nature Chem., 2019, 9, 11-16, b) S. Horike, S. Shimomura and S. Kitagawa, Nature Chem., 2009, 1, 659-704.

12 a) M. I. Gonzalez, M. T. Kapelewski, E. D. Bloch, P. J. Milner, D. A. Reed, M. R. Hudson, J. A. Mason, G. Barin, C. M. Brown and J. R. Long, J. Am. Chem. Soc., 2018, 140, 3412-3422, b) H. S. Cho, H. Deng, K. Miyasaka, Z. Dong, M. Cho, A. V. Neimark, J. K. Kang, O. M. Yaghi and O. Terasaki, Nature, 2015, 527, 503507.

13 Y. S. Li and S. Li, Spectrochimica Acta, 1994, 50A, 509-519.

14 H. M. Rietveld, J. Appl. Cryst., 1969, 2, 65-71.

15 B. Hourahine, B. Aradi, V. Blum, F. Bonafé, A. Buccheri, C. Camacho, C. Cevallos, M. Y. Deshaye, T. Dumitric, A. Dominguez, S. Ehlert, M. Elstner, T. vander Heide, J. Hermann, S. Irle, J. J. Kranz, C. Köhler, T. Kowalczyk, T. Kubar, I. S. Lee, V. Lutsker, R. J. Maurer, S. K. Min, I. Mitchell, C. Negre, T. A. Niehaus, A. M. N. Niklasson, A. J. Page, A. Pecchia, G. Penazzi, M. P. Persson, J. Rezác, C. G. Sánchez, M. Sternberg, M. Stöhr, F. Stuckenberg, A. Tkatchenko, V. W.-z. Yu and T. Frauenheim, J. Chem. Phys., 2020, 152, 124101 (1-19).

16 a) A. Heller and D. L. Williams, J. Phys. Chem. A, 1970, 74, 4473-4480, b) X. Peng, F. Song, E. Lu, Y. Wang, W. Zhou, J. Fan and Y. Gao, J. Am. Chem. Soc., 2005, 127, 4170-4171.

17 a) A. Douvali, A. C. Tsipis, S. V. Eliseeva, S. Petoud, G. S. Papaefstathiou, C. D. Malliakas, I. Papadas, G. S. Armatas, I. Margiolaki, M. G. Kanatzidis, T. Lazarides and M. J. Manos, Angew. Chem. Int. Ed., 2015, 127, 1671-1676, b) S. E. Henkelis, D. Rademacher, D. J. Vogel, N. R. Valdez, M. A. Rodriguez, L. E. Shea-Rohwer and T. M. Nenoff, ACS Appl. Mater. Interfaces, 2020, 12, 22845-22852.

18 a) Z. Hu, B. L. Deibert and J. Li, Chem. Soc. Rev., 2014, 43, 58155840, b) Y. Zhang, S. Yuan, G. Day, X. Wang, X. Yang and H-C. Zhou, Coord. Chem. Rev., 2018, 354, 28-45. 